\title{
How grim is pancreatic cancer?
}

\author{
Elroy Patrick Weledji, ${ }^{1}$ George Enoworock, ${ }^{2}$ Martin Mokake, ${ }^{1}$ Motaze Sinju' \\ ${ }^{1}$ Department of Surgery and Obstetrics and Gynaecology; and ${ }^{2}$ Department of Pathology, \\ University of Buea, Cameroon
}

\begin{abstract}
Pancreatic ductal carcinoma continues to be the most lethal malignancy with rising incidence. It is the fourth most common cause of cancer death in the western world due to its low treatment success rate. In addition, because of its rapid growth and silent course, diagnosis is often only established in the advanced stages. As one of the most aggressive malignancies, the treatment of this disease is a great challenge to clinicians. This paper reviewed the natural history of pancreatic cancer, the current clinical practice and the future in pancreatic cancer management.
\end{abstract}

\section{Introduction}

Pancreatic cancer is one of the most aggressive human malignancies, as $50 \%$ present with metastatic disease and $35 \%$ with locally advanced disease. ${ }^{1,2}$ It is the $13^{\text {th }}$ commonest cancer with 200,000 cases per year world-wide, 6000 cases per year in the UK and the fourth leading cause of cancer death in the Western world. ${ }^{2}$ There is an increasing incidence of this disease affecting 8-12 per 100,000 of the population per year. Whether this increased incidence is real or whether it reflects advances in diagnostic imaging is unknown. Its poor prognosis is manifested in an overall median survival of 4.4 months, and a 5 -year survival of $9.7 \%{ }^{3}$ Diagnostic problems arise because the symptoms are late and non-specific, there is no effective screening process and there is no specific high-risk group. Since conservative oncological therapies have failed to

Correspondence: Elroy Patrick Weledji, Department of Surgery and Obstetrics and Gynaecology, Faculty of Health Sciences, University of Buea, PO Box 126, Limbe, S.W. Region, Cameroon.

Tel.: +237.699922144.

E-mail: elroypat@yahoo.co.uk

Key words: Cancer; pancreas; prognosis; management.

Received for publication: 16 March 2016.

Revision received: 12 June 2016.

Accepted for publication: 29 June 2016.

This work is licensed under a Creative Commons Attribution NonCommercial 4.0 License (CC BY-NC 4.0).

@C Copyright E.P. Weledji et al., 2016

Licensee PAGEPress, Italy

Oncology Reviews 2016; 10:294

doi:10.4081/oncol.2016.294 show any benefit in long-term survival, resection remains the only modality of treatment offering any possibility of cure. ${ }^{4-7}$ Unfortunately, only $10-20 \%$ with head and less than $3 \%$ of body/tail cancers are candidates for resection. ${ }^{4,5}$ In the past 20 years, there is also only a modest increase in long-term survival with a median survival of 12 months, and 5-year survival rate of $15-26 \%$ after potentially curative resection. ${ }^{7}$ This has led to the development, of wider resections in the hope of increasing long-term survival, or more conservative approaches to improve the quality of survival. ${ }^{4,8}$ The role of these newer procedures is controversial and the Whipple's partial pancreaticoduodenectomy (PD) procedure is still favored by most surgeons. ${ }^{8-10}$ Traditional chemotherapy remains the standard treatment for advanced pancreatic cancer. Regimens like FOLFIRINOX (5-FU, leucovorin, irinotecan, and oxaliplatin) or gemcitabine and nab-paclitaxel have been used to palliate symptoms and prolong survival. ${ }^{11}$ This paper elucidates the grimness of pancreatic cancer and the potential for cure.

\section{Risk factors and genetics}

The etiology is largely unknown and the risk factors are listed in Table 1. Experimental studies have suggested that pancreatic carcinoma (PC) cell lines produce soluble factor(s) that can impair glucose metabolism and cause hyperglycemia. ${ }^{12-14}$ The $7-10 \%$ of pancreatic carcinoma are related to genetic factors with several well-defined genetic syndromes of familial pancreatic cancer (FPC) (Table 2). ${ }^{15,16}$ Although there are no specific genetic mutations identified for the majority of FPC (70\%), relatives of FPC kindred have a high risk of pancreatic cancer. ${ }^{17} \mathrm{~K}$-Ras gene mutations have been found in most pancreatic cancers. As a prediction of poor prognosis, the detection of K-ras mutations may be a useful prognostic factor for pancreatic cancer patients. K-Ras mutations are associated with a worse overall survival in pancreatic cancer patients, especially when mutations are detected in liquid biopsies or fresh frozen tumor tissue samples. ${ }^{18}$

Emerging studies show that cancer stem cells (CSCs) regulate several mechanisms underlying drug resistance, carcinogenesis, and metastases development in various types of cancer including pancreatic cancer. ${ }^{19,20}$ Recent studies dissected the role of microRNAs (miRNAs) and pancreatic CSCs on the modulation of pancreatic cancer etiology and progression, shedding light on their importance as potential therapeutic targets for pancreatic cancer. ${ }^{21,22}$

MicroRNAs are small noncoding RNAs involved in the regulation of gene expression at posttranscriptional level by binding to the 3 untranslated regions or the open reading frames of target genes. Oncogenic miR-21 lead to the repression of miRNA translation or to the degradation of target miRNAs. They modulate the proliferation and the chemoresistance of pancreatic cancer cells. ${ }^{23,24}$ In addition, there is a correlation between miR-21 expression and the clinical outcome of patients with pancreatic cancer through involvement of the PI3K/AKT pathway. ${ }^{20,24}$ 


\section{Pathophysiology and prognosis in ductal pancreatic cancer}

The majority (95\%) of pancreatic cancers develop as adenocarcinomas from the ductal cells of the exocrine pancreas. Of them $1 \%$ is of acinar origin, $1 \%$ is of non-epithelial origin and $3 \%$ are of uncertain cellular origin. ${ }^{25}$ It is associated with an accumulation of mutations with progressive morphological changes (Table 3$){ }^{26}$ The current model proposes a progression from normal cuboidal to low columnar epithelium through a series of lesions termed pancreatic intraepithelial neoplasia to invasive carcinoma. ${ }^{26,27}$ Prognosis in patients with pancreatic cancer depends on the tumor stage at time of diagnosis and tumor biology. The pattern of growth is characterized by early spread into local tissues, lymphatic and perineural sites, venous invasion and peritoneal metastases. Free cancer cells in the peritoneal cavity have been observed in $20-40 \%$ of cases, even in patients undergoing resection. ${ }^{28}$ Regional lymph-node metastasis occurred in $30 \%$ of patients with very small primary cancers and $64 \%$ of $\mathrm{T}_{1}$ primary cancer had lymph node involvement. ${ }^{28}$ Careful histological studies in a large series of resected pancreatic cancers revealed cancer dissemination in the lymph nodes in $89 \%$; lymph node metastases in $77 \%$, intrapancreatic neural invasion in $92 \%$ and a neural and nerve plexus invasion outside the pancreas in $45 \%$. ${ }^{29}$ Thus even though the surgeon may be able to offer resection to $>20 \%$ of patients with pancreatic cancer, the possibility of cure is gravely limited by the extent of early or occult micrometastases.

\section{Investigations}

Because of the poor prognosis care is taken not to over investigate or embark on treatment strategies based on unrealistic expectations of patients or their families. It is wise to use the fewest, least invasive and least expensive tests for patients not suitable for major resection. The investigations begin with baseline blood tests including a full blood count, renal function (urea and electrolytes), liver function, blood glucose, amylase and the tumor marker carbohydrate antigen (CA) 19-9. Many patients are anemic as a result of nutritional deficiency and chronic blood loss. The occurrence of frank gastrointestinal bleeding suggests the diagnosis of ampullary or duodenal carcinoma but stool examination usually reveals occult blood in patients with carcinoma of the pancreatic head. A rapid elevation of serum bilirubin and alkaline phosphatase on serial measurements of liver function will suggest a diagnosis of periampullary malignancy. Transaminase levels become elevated to a lesser degree reflecting injury to the hepatocytes as a result of unrelieved biliary obstruction. Blood glucose may be elevated. ${ }^{3} \mathrm{~A}$ transabdominal ultrasound should detect a pancreatic tumor and assess the liver, extrahepatic biliary tree and the pancreas. Its sensitivity of $70 \%$ falls to below $30 \%$ for tumors $<2 \mathrm{~cm}$ in diameter. ${ }^{30}$ A pancreatic protocol helical computed tomography (CT) scan of the abdomen would confirm the diagnosis and provide more detailed information for accurate staging. By assessing the tumor size, local extension/infiltration, intra/extrahepatic dilatation and metastasis it

Table 1. Risk factors for pancreatic adenocarcinoma.

\begin{tabular}{ll}
\hline Age & $80 \%$ aged $70-80$ years; $1 \%<40$ years \\
Sex & Women $>$ men \\
\hline Race & African Americans $>$ whites $>$ Asian Americans \\
Smoking, alcohol, coffee, high fat high protein low fiber diet & $10 \times$ relative risk if $>2$ packs/day \\
\hline Chronic pancreatitis & 5 -15-fold $\uparrow$ risk \\
Hereditary pancreatitis & $50-70$-fold $\uparrow$ risk \\
Diabetes mellitus & $2 \times$ relative risk diabetic onset $>3$ years before diagnosis \\
& $3 \times$ relative risk diabetic onset $>2$ years before diagnosis \\
\hline & $2-5 \times$ the relative risk: \\
Abdominal surgery & Prior partial gastrectomy, cholecystectomy \\
Occupational & Napthylamine, ethyl dichloride, benzidine, metal-gas workers, chemists \\
Hereditary (4-16\%) & Familial: \\
& 2 relatives affected, $18 \times \uparrow$ risk \\
& 3 relatives affected, $57 \times \uparrow$ risk \\
\hline
\end{tabular}

Table 2. Syndromic familial pancreatic cancer.

\begin{tabular}{lc} 
Genetic syndrome & Germline mutation \\
Hereditary pancreatitis & PRSS1 \\
HNPCC (Lynch 11) & hMSH2, hMLH1 \\
\hline Familial breast cancer & BRAC-2 \\
FAMMM syndrome & P16 \\
\hline FAP & APC \\
Peutz-Jeghers syndrome & STK11/LKB \\
\hline
\end{tabular}

HNPCC, hereditary non-polyposis colorectal cancer; FAMMM, familial atypical multiple mole melanoma; FAP, familial adenomatous polyposis.
Table 3. Frequency of mutations in pancreatic cancer.

\begin{tabular}{lc} 
Mutation & Incidence (\%) \\
K-ras 2 & 95 \\
P16/CDKN2A & 80 \\
P21 & $75-85$ \\
\hline TP53 & $50-75$ \\
Cyclin D1 & 95 \\
DPC4/MADH4 & 55 \\
\hline Telomerase & 95 \\
BRCA-2 & $7-10$ \\
\hline LKB1/STK11, MKK4, TGF $31 / 11$, RB1 & 5 \\
\hline
\end{tabular}


would predict resectability in $80-90 \%$. The CT criteria for resectability are: i) no extrapancreatic disease; ii) no direct tumor extension to the coeliac axis or superior mesenteric artery (SMA); and iii) a patent SMA/portal vein (SMV-PV) confluence. ${ }^{31}$ Magnetic resonance cholangio-pancreatography and magnetic resonance angiography (MRA/) if suspected vascular invasion on CT would delineate abnormal pancreatic and vascular anatomy. The positron emission tomography scan excludes metastatic disease. ${ }^{32}$ Endoscopic ultrasonography (EUS) would assess tumor size, vascular involvement, local invasion and nodal status with or without fine needle aspiration cytology. ${ }^{33}$ The greatest weakness of EUS is its inability to detect small peritoneal deposits and liver metastases which contribute to its overall accuracy of only $66 \%$ in the American Joint Committee on Cancer (AJCC) TNM staging of pancreatic cancer. ${ }^{34}$ Further information may be obtained with endoscopic retrograde cholangiopancreatography (ERCP), which has better sensitivity and specificity for the diagnosis of pancreatic abnormalities than CT and may enable the relief of obstructive jaundice by placement of a stent into the bile duct which some authors regard as standard preparation for resection. ${ }^{35,36}$ With a combination of these methods approximately $90 \%$ of pancreatic cancers can be detected preoperatively. ${ }^{36}$

\section{Is there a role for pre-operative biliary drainage?}

Pre-operative biliary drainage (endoscopic or transhepatic) remains controversial. Clinical trials have failed to show a reduction in postoperative morbidity and mortality. ${ }^{35,36}$ The reduced complications from resolution of jaundice are offset by more inflammatory tissue at surgery and the higher rate of biliary sepsis from contamination and post-operative infective complications. ${ }^{36,37}$ Preoperative drainage can also often delay definitive treatment and therefore many surgeons prefer to proceed straight to pancreatectomy and reserve biliary drainage for those with evidence of cholangitis, impaired renal function or prolonged jaundice with high bilirubin concentration ( $>100 \mathrm{umol} / \mathrm{L}$ ). ${ }^{36}$ In cases with tumor invasion into the distal part of the common bile duct, the ERCP procedure is not always possible. Therefore, the possibility of percutaneous transhepatic cholangiostomy, may be qualified as palliative intervention in the case of unresectable tumor. ${ }^{36}$

\section{Pre-operative staging}

Preoperative staging is based on the AJCC TNM staging system (Table 4). The Japanese Pancreatic Society (JPS) classification is similar to TNM except lymph node groups are clearly defined. Staging laparoscopy, intraoperative liver US and peritoneal cytology may improve accuracy of staging and avoid unnecessary surgery in patients with occult peritoneal disease in up to $10 \% .^{38}$ The criteria for unresectability at staging laparoscopy are: i) histologically confirmed hepatic, serosal, peritoneal, or omental metastases; ii) extrapancreatic extension of tumor, e.g., mesocolic involvement; iii) histologically confirmed coeliac or high portal node tumor involvement; iv) invasion or encasement of the coeliac axis, hepatic artery or SMA., 89

\section{Is there a role for biopsy?}

Operative biopsy of pancreatic tumor is rarely necessary. Pre-operative ERCP biopsy, brushings, cytology or endoluminal US will differentiate chronic pancreatitis from carcinoma of pancreas and at least a fistula may occur but into the duodenum following an endoscopic biopsy. US or CT-guided fine-needle biopsy is avoided in patients with potentially resectable tumors as it can cause peritoneal seeding and spread of the tumor. ${ }^{40}$ In addition, there is a high rate of false negativity, especially if the tumor is very small or with a fibrotic reaction. However, whether benign or malignant, resection of an obstructive pancreatic lesion is beneficial to the patient. Intraoperative trucut biopsy of an inoperable pancreatic cancer at laparotomy is justifiable for documentation. ${ }^{36}$

\section{Criteria for resectability and oncological standards of surgical resection}

There is a growing consensus on the radiological definitions of resectable, borderline resectable and unresectable, and the National Comprehensive Cancer Network in the USA has endorsed a modified consensus from the Americas Hepato-Pancreato-Biliary Association,

Table 4. American Joint Committee on Cancer (AJCC) TNM staging of pancreatic cancer.

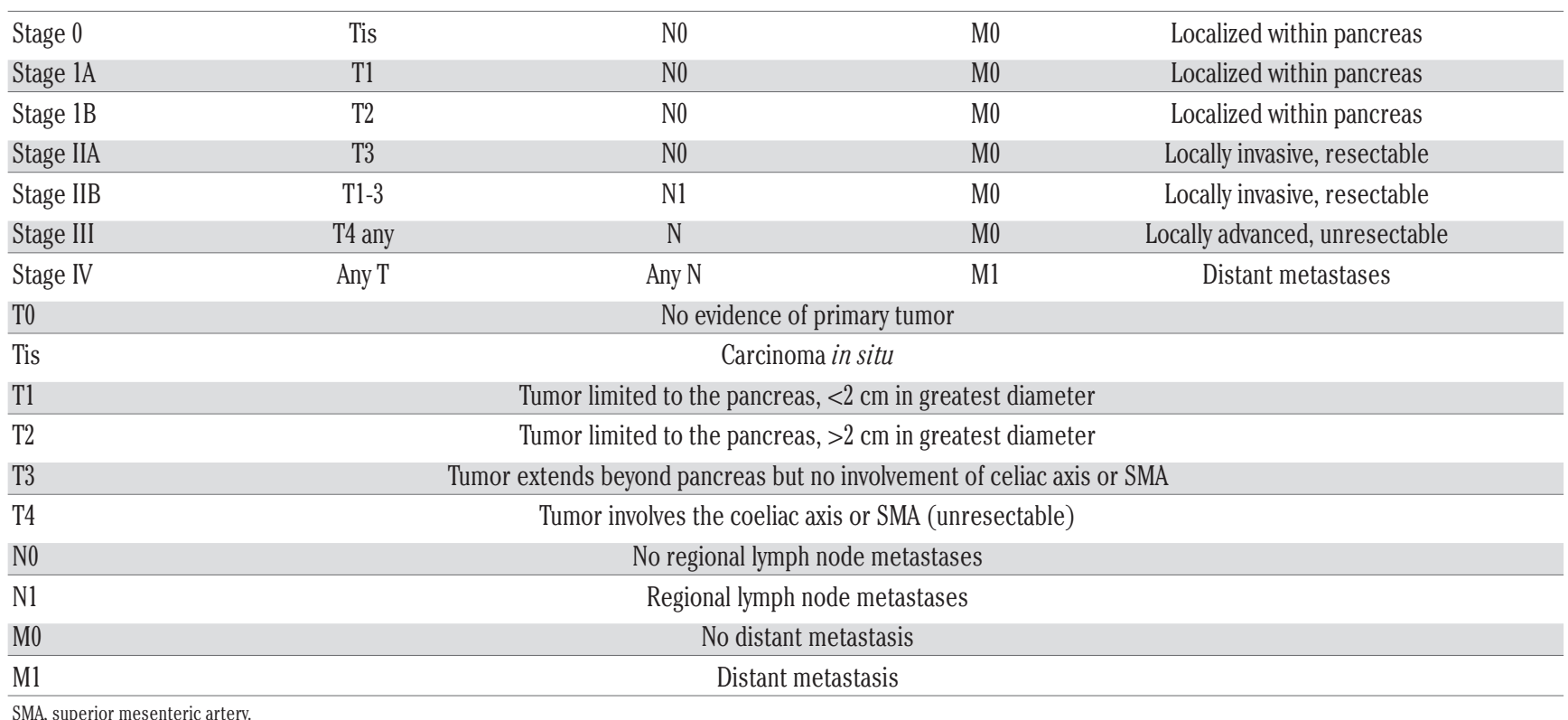


the Society of Surgical Oncology and the Society for Surgery of the Alimentary Tract. ${ }^{41} \mathrm{~A}$ tumor is potentially resectable if it can be technically removed with negative margins (R0 resection) without compromising the vascular supply to the liver (hepatic artery) or small bowel (superior mesenteric artery). Involvement of adjacent organs (e.g, duodenum or transverse colon), regional lymph nodes, portal vein (partial involvement), gastroduodenal artery, are not contraindications to resection, as these structures can be removed en bloc with the tumor to achieve an R0 (no tumor cells within $1 \mathrm{~mm}$ ) resection. ${ }^{4-6,8}$ A tumor is unresectable in the presence of: major comorbidity, metastatic disease (including involved lymph nodes out with the resection field, locally advanced disease with extrapancreatic involvement, superior mesenteric artery or coeliac artery involvement, and main portal venous occlusion/thrombosis. PV encasement from external compression with occlusion and thrombosis is a contraindication to resection because arterial involvement is likely to co-exist. ${ }^{8,42} \mathrm{An} \mathrm{R} 0$ resection for ductal pancreatic cancer must include an N1 and N2 lymph node dissection, perivascular connective tissue dissection and a standardized retroperitoneal soft-tissue dissection. ${ }^{43}$ Favorable prognostic features include negative resection margin, negative lymph nodes, well/moderately differentiated carcinoma, primary $<2 \mathrm{~cm}$ diameter and no perineural or lymphovascular invasion..$^{4}$ Patients with negative lymph nodes have significantly higher 3 - and 5-year survival rates than patients with positive lymph nodes. ${ }^{45}$ However, most $\mathrm{R} 0$ resections (70\%) are actually $\mathrm{R} 1$ (one or more tumor cells within $1 \mathrm{~mm}$ ) resections as a result of microscopically incomplete resection and the biological features of the tumor such as frequent neural invasion. This highlights the importance of specialized surgeons and pathologists in the treatment of this condition. ${ }^{44,45}$

\section{Extended vs standard lymphadenectomy}

Several centers have reported a survival benefit with extended (radical) lymphadenectomy (standard plus resection of lymph nodes along arterial supply including an en-bloc lymphadenectomy of the hepatoduodenal ligament) compared with standard lymphadenectomy (resection of peripancreatic, periduodenal and perigastric lymph nodes) ${ }^{46,47}$ However data from randomized control trials (RCTs) and a meta-analysis did not show any benefit, but a potentially increased morbidity with extended lymphadenectomy. Extended lymphadenectomy might thus not be recommended outside of adequately powered RCTs or specialist centers as it is also apparent that survival in patients with an R0 resection including N1 and N2 lymph node dissection is only marginally longer than in those with an R1 resection provided adjuvant chemotherapy is used. ${ }^{48,49}$ However, the surgical dexterity and precision of robotic surgery will facilitate extended lymphadenectomy with minimal morbidity. ${ }^{50}$

\section{Portal vein resection}

Historically, portal vein involvement was a contraindication to resection. However, PV or SMV encasement is now considered to be related to tumor location, rather than biological behavior. ${ }^{40,41}$ Due to the unsatisfactory results of the standard Whipple's (PD) resection and total pancreatectomy, Fortner in 1973 described regional pancreatectomy in order to achieve a negative resection margin and improve long-term survival. $^{51}$ This consists of an en bloc resection of the tumor with an adequate peripancreatic soft tissue margin, regional lymph nodes with portal vein resection (Type I) or resection/reconstruction of a major artery (Type II). The reconstruction can very often be achieved by a direct endto-end anastomosis of the venous remnants or the resected PV replaced if necessary with Dacron graft or saphenous interpositional vein graft. Early results were poor (morbidity 67\%; mortality 23\%) with 3\% 3-year survival. Portal vein resection was associated with longer operating time, increased blood loss, increased perioperative morbidity and mortality as compared to standard PD (Whipple's). Regional pancreatectomy is now associated with $<4 \%$ mortality, $26 \% 5$-year survival and reduced morbidity in high volume centers due to: i) advances in radiological and surgical techniques; ii) improved staging; iii) better patient selection; and iv) adjuvant chemotherapy ${ }^{52,53} \mathrm{PD}$ with $\mathrm{PV}$ resection should only be performed at centers with expertise in complex pancreatic surgery and only if an R0 resection can be achieved. ${ }^{54-56}$ However, PD with PV resection has similar survival compared to standard PD (in absence of SMA or coeliac axis involvement). Resection margins (R0 vs R1/R2) and lymph node status are more important than portal vein involvement per se in terms of long-term survival. $3,8,9$

\section{Management options}

\section{Role of multimodal treatment in pancreatic cancer}

Surgery remains the only chance of cure for pancreatic cancer, but only $15-25 \%$ of patients present with resectable disease at the time of primary diagnosis. Important goals in clinical research must therefore be to allow early detection with suitable diagnostic procedures, to further broaden operation techniques and to determine the most effective perioperative treatment with either chemotherapy and/or radiation therapy. Multimodal treatment including adjuvant chemotherapy and radiotherapy for resectable pancreatic cancer was superior to conventional surgical treatment with a four-year survival rate of above $40 \%$ in the treatment group. ${ }^{57}$ Surgical resection without adjuvant treatment produces favorable five-year survival only in patients with early stage I pancreatic cancer. ${ }^{58-63}$ Tsuchiya et al. reported a 5-year survival rate of only $30 \%$ in patients with stage II cancer. ${ }^{58}$ The results of the Gastrointestinal Tumor Study Group using adjuvant radio-chemotherapy following resection of pancreatic cancer revealed a median survival of 21 months in the group randomized to treatment compared to 10.9 months in the control group. ${ }^{59}$ The two-year actuarial survival was $46 \%$, and $18 \%$ in the control group and the five-year probability survival in the treatment group was above 20\%. The European Study Group for Pancreatic Cancer (ESPAC-1-Trial) showed a 5-year survival benefit of adjuvant chemotherapy with gemcitabine for 6 months to patients with lymph node involvement ( $24 \%$ vs $9 \%$ 5-year survival). ${ }^{60}$ Locoregional chemotherapy administered via the hepatic artery of a regimen comprising 5-fluorouracil (3FU) folinic acid, mitoxantrone and cis-platinum for 6 months in Stage II and III cancers showed improvement of the median survival from 9.3 to 16 months compared to stage 1 cancers with resection only. ${ }^{61-63}$ While gemcitabicin is the main drug in the chemotherapy, recent studies indicate the effectiveness of combination schemes, in particular, nab-paclitaxel plus gemcitabine over gemcitabine monotherapy or when FOLFORINOX fails for metastatic pancreatic cancer. ${ }^{64,65}$ Additionally, therapies with a broader mechanism of action are emerging (stroma depletion, immunotherapy, anti-inflammation), raising hopes for more effective adjuvant and neoadjuvant treatment concepts, especially in the context of borderline resectability. ${ }^{63}$

\section{Surgical options}

The surgical options entail: i) a realist approach of positive patient selection for radical resection or palliative stent or surgical bypass; ii) an activist approach of regional pancreatectomy plus transplantation; and iii) the nihilist approach of palliative stenting or bypass. The main factors in determining optimal treatment of pancreatic carcinoma is the tumor stage and the patient's fitness for major surgical resection (Table 4).$^{4-8}$ Surgical resection does not improve survival in patients with locally advanced or metastatic disease (Table 5 and Figure $1^{63}$ ).,4

\section{Resectable disease (Stage 1-11)}

Resectable tumors are $<4 \mathrm{~cm}$ in diameter and confined to the pancreas with no local invasion or metastases. ${ }^{3,4}$ Preoperatively, coagulopa- 
thy should be corrected by the parenteral administration of vitamin K. To prevent the development of post-operative renal failure patients should be well hydrated and any electrolyte imbalance rectified. Prophylactic antibiotics are prescribed routinely, and some patients may benefit from preoperative parenteral nutrition. Patients with tumor in head of pancreas may benefit from: i) Whipple's PD which is the only hope for cure in $<15 \%$; ii) pylorus preserving pancreaticoduodenectomy; iii) PD with en bloc vascular resection and reconstruction (regional pancreatectomy).$^{6,9}$ Although very few ductal carcinomas of the body and tail are resectable (3\%) due to the advanced nature of these tumors at presentation these patients may benefit from distal pancreatectomy or total pancreatectomy for the rare slow-growing malignant tumors, which include cystadenocarcinoma and papillary-cystic neoplasm. ${ }^{3-9}$ Currently, minimally invasive techniques (laparoscopy and robotic techniques) are used for surgical treatment of early forms of pancreatic cancer with the advan- tages of a high degree of eligibility as well as significant reductions in length of stay, wound infections, and pancreatic fistula. The overall survival after laparoscopic PD is similar to open PD. ${ }^{66}$ Robotic-assisted PD is safely feasible in selected patients and the results in pancreatic cancer are encouraging but deserve further investigation..$^{50}$

The poor survival rate associated with surgery alone for early-stage PC has led to adjuvant therapy becoming the standard of care after resection in an effort to prolong survival. Neoadjuvant and adjuvant chemotherapy or chemoradiotherapy improves the chance of cure for early PC. ${ }^{59-61}$ CT or magnetic resonance imaging (MRI) is used in the oncological follow-up of patients with resections for pancreatic carcinoma. It is started 6 months after the operation and in 6 -month intervals for 2 years postoperatively. Then yearly intervals seem to be sufficient. In addition the serum levels of carcino-embryonic antigen (CEA) and CA 19-9 are also evaluated. ${ }^{3,4,7,8,67}$

Table 5. Management options.

\begin{tabular}{|c|c|c|}
\hline Resectable diseases (Stages I-II) & $\begin{array}{l}\text { Locally advanced unresectable disease } \\
\text { (Stage III) }\end{array}$ & $\begin{array}{l}\text { Metastatic disease } \\
\text { (Stage IV) }\end{array}$ \\
\hline \multirow[t]{3}{*}{$\begin{array}{l}\text { Surgical resection (with or without adjuvant } \\
\text { chemotherapy or chemoradiotherapy) }\end{array}$} & $\begin{array}{l}\text { Neoadjuvant chemoradiotherapy } \\
\text { then restaging CT with or without resection } \\
\text { if down-staged }\end{array}$ & Palliative chemotherapy (gemcitabine) \\
\hline & $\begin{array}{c}\text { Palliative chemotherapy: } 5 \text {-FU, folinic acid, } \\
\text { and gemcitabine } \\
\text { Palliative chemoradiotherapy }\end{array}$ & $\begin{array}{c}\text { Best supportive care } \\
\text { Pain control } \\
\text { Consider celiac plexus block }\end{array}$ \\
\hline & Palliation of biliary and gastric outlet obstruction & Palliation of biliary and gastric outlet obstruction \\
\hline
\end{tabular}

CT, chemotherapy.

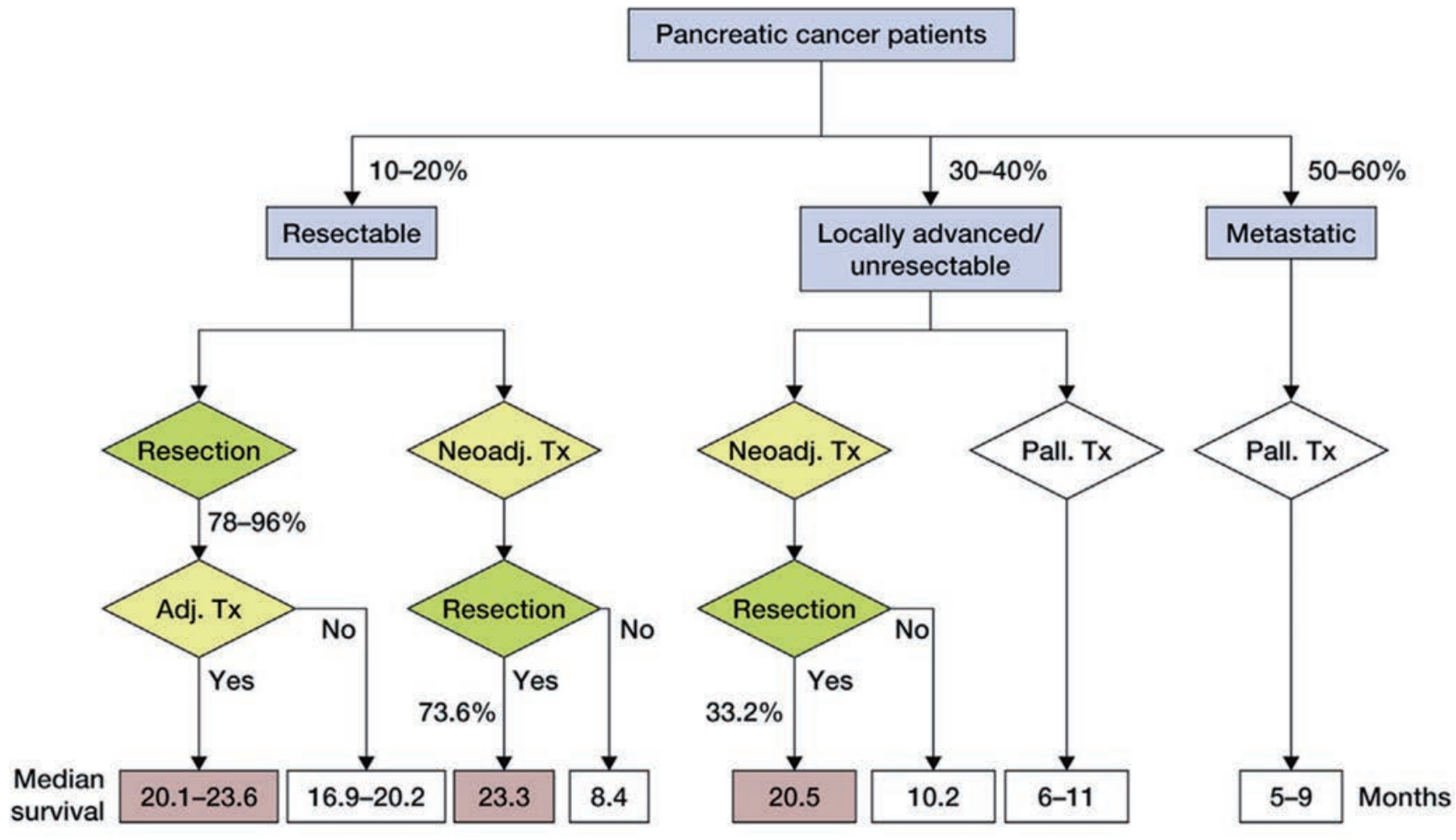

Figure 1. Summary of survival and resection percentages for patients with pancreatic cancer. Neoadj., neoadjuvant; Tx, treatment; Pall., palliative; Adj., adjuvant. Reproduced with permission from Gillen et al., $2010 .^{63}$ 
Locally advanced (unresectable) disease (Stage 111)

Of patients $35 \%$ with pancreatic cancer present with locallyadvanced disease. ${ }^{3,4}$ The management options are: i) neoadjuvant chemoradiotherapy then re-staging CT and resection if down-staged as the best palliation is surgical resection; ii) palliative chemotherapy with 5-FU, folinic acid, and gemcitabine; iii) palliative chemoradiotherapy; and iv) palliation of biliary and gastric outlet obstruction.,3,68

\section{Palliative surgery}

It is recommended that biliary bypass is combined with gastric bypass (double bypass) in most patients with unresectable cancer of the pancreatic head. The mortality rate of biliary bypass is not increased by adding a gastroenterostomy. Following biliary bypass alone, $17 \%$ of patients will develop duodenal invasion and obstruction as the tumor enlarges requiring subsequent gastric bypass which carries an average mortality rate of $22 \%{ }^{68}$ However, while surgical bypass is an effective means of relieving biliary obstruction (hepaticojejunostomy) and avoiding duodenal obstruction (gastrojejunostomy), it is not without risk in this elderly ill patient population. The mean operative mortality of bypass in one series was no less than 19\% (range 4-30\%), and it was clear that much depended on patient selection. ${ }^{69}$ Given that the mean duration of survival following bypass is only 5.4 months, nonoperative methods of stenting (endoscopic, percutaneous) have been embraced so enthusiastically, almost to the point at which surgical bypass is reserved for patients in whom stenting is technically not possible. ${ }^{70}$ Despite the success of stenting and its lower early mortality, stent blockage remains the major drawback with resulting cholangitis and return of jaundice and pruritus. ${ }^{71,72}$ However, surgical palliation may enjoy a comeback in selected patients, particularly now that biliary-enteric and gastric bypass can be achieved by laparoscopic means with the additional benefit of unequivocal assessment of resectability. ${ }^{4,8,39,66}$ Multivariate analysis of patients with unresectable pancreatic cancer revealed age, stage of disease serum albumin, serum C-reactive protein (CRP) levels as independent predictors of duration of survival. The presence of an acute phase response (C-reactive protein level) was the single most significant independent predictor with a median survival of 66 days with $\mathrm{CRP}<10 \mathrm{mg} / \mathrm{L}$ compared to 222 days in those with no acute phase response. ${ }^{73}$ Thus, the importance of cachexia as a cause of early death in pancreatic cancer and the elucidation of the relationship between cytokine profile, acute phase response and resting energy expenditure.

\section{Metastatic disease (stage IV)}

Of patients $50 \%$ with pancreatic cancer present with metastatic disease and the majority are beyond significant palliation.3,48 Management options of metastatic disease include palliative chemotherapy (gemcitabine), best supportive care, pain control with consideration of coeliac plexus block and palliation of biliary and gastric outlet obstruction. For patients with metastatic disease, the primary goals of treatment are palliation and improved survival, yet effective treatments for this population are limited, leading to extremely poor survival rates (5-9 months). ${ }^{3}$ Gemcitabine has been the standard treatment for metastatic disease, primarily due to its effect on symptoms and favorable toxicity profile rather than a significant effect on survival. The combination of gemcitabine with oxaliplatin may confer an additional but small survival benefit in patients with good performance status and a younger age. ${ }^{74}$

\section{The surgeon's dilemma}

With the exception of true periampullary tumors, where radical resection may definitely offer the prospect of long-term survival, carcinoma of the head of the pancreas is generally considered incurable and management directed towards adequate palliation. ${ }^{75-79}$ There are however, genuine arguments for and against surgical resection.

\section{Arguments for surgical resection}

The arguments for surgical resection are as follows: i) carcinoma of the pancreas is increasing in incidence and the use of modern diagnostic imaging techniques e.g., US, CT, MRI can pick up tumors at an early stage; ii) even though the chances of cure are $<10 \%$, the only hope for cure is by surgical resection; ${ }^{6}$ iii) if we exclude all patients from consideration for surgery we may exclude patients suffering from carcinoma of distal common bile duct, the duodenal mucosa and ampulla of Vata having a 5 year survival rate of $30 \% ; 2,80$ iv) the operative mortality has fallen to $5 \%$ or less in experience hands; ${ }^{4,6,8}$ v) although it is considered a disease of the elderly more than $40 \%$ of men and $35 \%$ of women present under the age of 70 years (Figure 2). ${ }^{2}$

\section{Arguments against surgical resection}

The arguments against surgical resection are: i) pancreatic carcinoma usually has an insidious presentation and physical signs of metastatic spread are commonly present at initial consultation; $81-83$ ii) it is a disease of elderly patients and $50 \%$ are $>72$ years. Many are unfit, weak, emaciated and suffer from other concomitant medical conditions. Endoscopic bypass is all that can be offered; 68 iii) bypass procedures are all that can be achieved in the vast majority; 69 iv) an unsuccessful resection for a carcinoma can result in a high mortality, a very high morbidity and an extremely costly period of treatment for the patient. $8,51,52$

\section{Why persistently poor long-term results after pancreatic resection?}

The answer largely lies in the biological nature of pancreatic cancer, which demonstrates aggressive local invasion, and metastases during

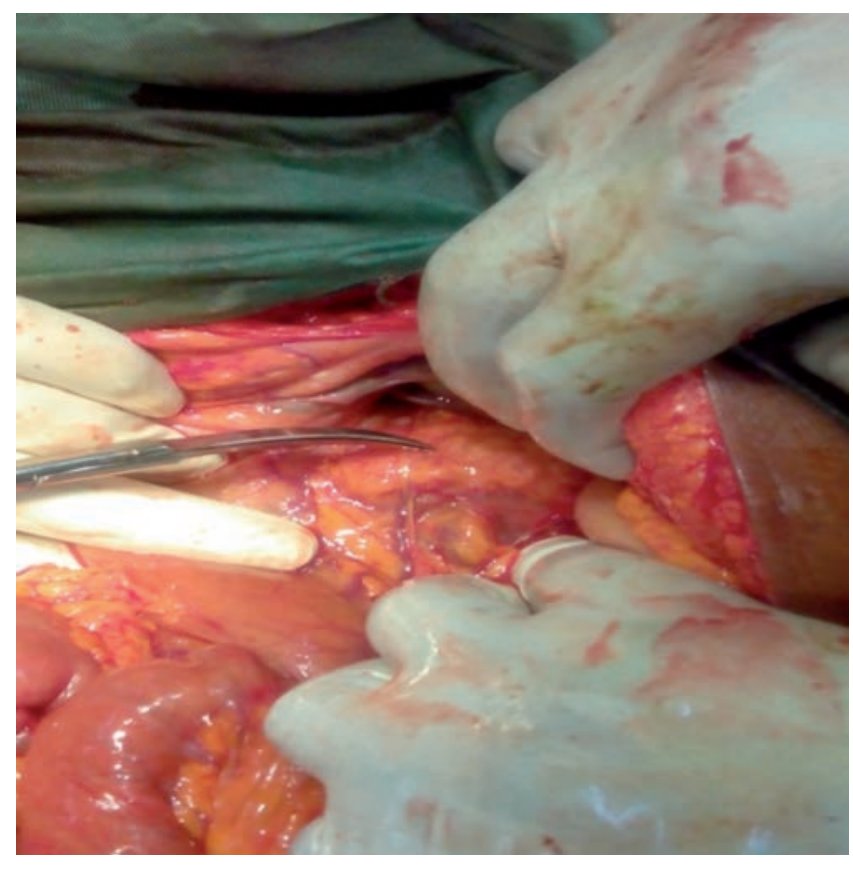

Figure 2. Locally advanced pancreas cancer in a 70-year-old African woman requiring double bypass procedure in a low-resourced setting (note: the peripancreatic fat has been effaced by the tumor). With permission from the patient in our Surgical Unit. 
early development. Over $80 \%$ of patients have positive regional lymph nodes or distant metastases at the time of diagnosis. ${ }^{77,78}$ Studies have revealed that even in small pancreatic tumors, which have not spread through the pancreatic capsule and with a diameter of less than $2 \mathrm{~cm}$, there are positive para-aortic lymph nodes in $40 \%$ and therefore classified as stage II disease. ${ }^{81,82}$ Thus small tumor size cannot automatically be equated with early tumor stage. The biological grade is most important. Pancreatic cancer extends preferentially along lymphatic channels and nerves and readily invades blood vessels. The close interaction of tumor cells and neural structures is mediated by molecular signals including the neural cell adhesion molecules (NCAM) which facilitates invasion and metastases..$^{83,84}$ Nerves have been found to express these factors in abundance, thus providing a positive growth stimulus for pancreatic cancer cells. ${ }^{83}$ These may partially explain the early spreading nature and the fact that at least $50 \%$ of cases have a local recurrence within the tumor bed following resection..$^{79,85}$ The implication is that by targeting NCAM, cytotoxic drugs may be the delivered directly to the pancreatic cancer cells with better efficacy and less systemic side effects than systemic chemotherapy.

\section{Use of tumor associated antigens in diagnosis and follow-up}

The most widely used marker is CA 19-9 antigen as it is expressed in $86 \%$ of pancreatic cancers with a sensitivity of $89 \%$ compared to the sensitivity of $37 \%$ with CEA. $70 \%$ of patients with a tumor $<4 \mathrm{~cm}$ already show elevated serum levels. ${ }^{86}$ Additionally CA 19-9 levels correlate with prognosis as it is more significantly lower in small resectable tumors than in larger ones. However, its sensitivity is not high enough for the primary diagnosis of pancreatic cancer. ${ }^{87} \mathrm{CA} 19$ 9 is elevated in patients with non-malignant diseases, such as chronic pancreatitis or obstructive jaundice of various origins and in smoking. Its determination has a high clinical value if a CT scan indicate a pancreatic cancer and in the follow-up of patients following resection. If the CA 19-9 level returns to normal after tumor resection and increases during follow-up, then cancer relapse is extremely probable. ${ }^{88}$

\section{The future}

Progress in identifying new therapies has been hampered by the genetic complexity of the disease with each tumor cell carrying an average of 63 mutations, and the lack of prognostic markers. ${ }^{89}$ Most alterations occur with very low frequency and so are challenging to exploit therapeutically. The future lies on the better understanding of the molecular oncology of pancreatic cancer, which entails the genetics and the pathophysiology of metastasis of pancreatic cancer. About $75 \%$ of human pancreatic adenocarcinomas have acquired a mutation in codon 12 of the $K$-ras gene and there could be a role for biological therapy countering the effects of specific mutant oncogenes. ${ }^{90}$ Inactivation of tumor-suppression genes occurs frequently during the development of cancers with loss of function of the $p 53$ gene in $60 \%$ of primary ductal adenocarcinoma of the pancreas..$^{91}$ Replacement of tumor suppressor functions may therefore be a useful therapeutic strategy. Several receptor tyrosine kinases, including epidermal growth factor receptor family (EGF-R, erbB-2, erB-3) are frequently over-expressed in pancreatic cancer and involved in signal-transduction pathways. ${ }^{90,91}$ Inhibition of signal transduction may play a role in preventing metastases. Although the results of initial trials with EGFR inhibitor (erlotinib) in unselected patients have been disappointing, the agents may be useful in KRAS-mutant p53 wild type tumors which occurs in $15 \%$ of patients. ${ }^{92}$ New-onset diabetes may also be a potential clue to the early diagnosis of pancreatic cancer. ${ }^{12}$ Genetic data have been interpreted to suggest that development of invasive disease from precursor lesions occurs over a considerable length of time (17 years on average), with death following after 2-3 years, highlighting the importance of identifying early diagnostic markers of pre-invasive pancreatic cancer. ${ }^{93,94}$ The recent major break- through is in the identification of early protein markers (cancer exosomes) that may provide early diagnosis and represent a valid screening test. ${ }^{95}$ This would lead to early surgical intervention with a better chance of curing this essentially incurable disease. As familial aggregation and genetic susceptibility play a role in as many as $10 \%$ of patients with pancreatic cancer, screening of first-degree relatives of this and other high risk groups for these protein markers would allow early intervention and improve longterm survival. ${ }^{17}$

\section{Immunotherapy and vaccination}

Immune therapy is changing the current treatment paradigm for malignancy, especially with the recent development of antibodies that can modulate immune checkpoint pathways. Immunotherapy to treat pancreatic cancer is a promising approach due to its low toxicity and potential for creating life-long immune response. Multiple large phase III trials using simple vaccination strategies have failed to modulate the immune response in pancreatic cancer. However novel strategies with whole cell vaccines using hyperacute rejections (algenpantucel-L) immunotherapy demonstrated $62 \%$ and $86 \% 12$-month disease free survival and overall survival in resected pancreatic cancer patients. Combination of whole cell vaccine GVAX and mesothelin-secreting vaccine CRS-207 demonstrated an overall survival benefit in metastatic refractory pancreatic cancer patients. ${ }^{96}$

Anti-Gal is the most abundant natural antibody in humans, comprising about $1 \%$ of immunoglobulins. The anti-Gal ligand is a carbohydrate antigen called $\alpha$-gal epitopes with the structure Gal $\alpha 1$ $3 \mathrm{Gal} \beta 1-4 \mathrm{GlcNAc}-\mathrm{R}$. It is exploited in cancer vaccines to increase the immunogenicity of antigen-presenting cells (APCs). As cancer cells or pancreatic ductal adenocarcinoma cells (PDAC) tumor lysates are processed to express $\alpha$-gal epitopes, vaccination with these components results in in vivo opsonization by anti-Gal IgG in PDAC patients. The Fc portion of the vaccine-bound anti-Gal interacts with Fc receptors of APCs, inducing uptake of the vaccine components, transport of the vaccine tumor membranes to draining lymph nodes, and processing and presentation of tumor-associated antigens (TAAs). Cancer vaccines expressing $\alpha$-gal epitopes elicit strong antibody production against multiple TAAs contained in PDAC cells and induce activation of multiple tumor-specific T cell..$^{97}$

A pancreatic tumor-specific biomarker characterized in humans and mice as an immunogenic onco-glycoprotein is efficient in dendritic cell vaccination. Thus murine DC, loaded with pancreatic tumor-specific glycoepitope C-ter-J28+, induces efficient anticancer adaptive immunity and represents a potential adjuvant therapy for patients afflicted with PDAC. 98

\section{Conclusions}

Pancreatic ductal adenocarcinoma is still a disease with a very poor prognosis. It is genetically very complex with a high diversity of mutations compared with other cancers. Early diagnosis with the new protein markers may lead to early intervention and better prognosis. The main surgical goal in performing an $\mathrm{R} 0$ resection facilitated by improved staging and patient selection would result in hospital mortality of $<5 \%$ in specialist centers. As pancreatic carcinoma is largely resistant to standard chemotherapy, consideration of multimodal treatment including immunotherapy is necessary. 


\section{References}

1. Jemal A, Bray F, Center MM, et al. Global cancer statistics. CA Cancer J Clin 2011;61:69-90.

2. Siegel R, Naishadham D, Femal A. Cancer statistic, 2012. CA Cancer statistics, 2012. CA Cancer J Clin 2012;62:10-29.

3. Wolfgang CL, Herman JM, Laheru DA, et al. Recent progress in pancreatic carcinoma. CA cancer J Clin 2013;63:318-48.

4. Ozawa F, Friess H, Kunzli B, et al. Treatment of pancreatic cancer: the role of surgery. Dig Dis 2001;19:47-56.

5. Johnson CD, Schwall G, Flechtenmacher J, Trede M. Resection for adenocarcinoma of the body and tail of the pancreas. Br J Surg 1993; 80:1177-7.

6. Wagner M, Redaelli C, Lietz M, et al. Curative resection is the single most important factor determining outcome in patients with pancreatic adenocarcinoma. Br J Surg 2004;91:586-94.

7. Conlon KC, Klimstra DS, Brennan MF. Long-term survival after curative resection for pancreatic ductal adenocarcinoma. Clinicopathologic analysis of 5-year survivors. Ann Surg 1996;223: 273-9.

8. Michalski CW, Weitz J, Büchler MW. Surgery Insight: surgical management of pancreatic cancer. Nature Clinical Practice Oncology 2007;4:526-35.

9. Liu SL, Friess H, Kleeff J, et al. Surgical approaches for resection of pancreatic cancer: an overview. Hepatobiliary Pancreatic Dis Int 2002;1:118-2.

10. Itani KMF, Colman RE, Meyers WC, Akwardi OE. Pylorus - preserving pancreato-duodenectomy. A clinical and physiologic appraisal. Ann surg 1986;204:655.

11. Sinn M, Bahra M, Denecke T, et al. Perioperative treatment options in resectable pancreatic cancer - how to improve long-term survival. World J Gastrointestinal Oncol 2016;8:248-57.

12. Pannala R, Basu A, Peterson GM, et al. New-onset diabetes: a potential clue to the early diagnosios of pancreatic cancer. Lancet Oncol 2009;10:88-95.

13. Magruder JT, Elahi D, Anderson DK. Diabetes and PC: chicken or egg? Pancreas 2011;22:189-97.

14. Basso D, Brigaro L, Veronesi A, et al. The PC cell line MIA PaCa2 produces one or more factors able to induce hyperglycemia in SCID mice. Anticancer Res 1995;15:2585-8.

15. Permuth-Wey J, Egan KM. Family history is a significant risk factor for pancreatic cancer: results from a systematic review and metaanalysis. Fam Cancer 2009;8:109-17.

16. Tersmette AC, Petersen GM, Offerhaus GJ, et al. Increased risk of incident pancreatic cancer among first-degree relatives of patients with familial pancreatic cancer. Clin Cancer Res 2001;7:738-44.

17. Shi C, Hruban RH, Klein AP. Familial pancreatic cancer. Arch Pathol Lab Med 2009;133:365-74.

18. Tao LY, Zhang LF, Xiu DR, et al. Prognostic significance of K-ras mutations in pancreatic cancer: a meta-analysis. World J Surg Oncol 2016;14:146.

19. Bimonte S, Barbieri A, Leongito M, et al. The role of miRNAs in the regulation of pancreatic cancer stem cells. Stem Cells Int 2016; 2016:8352684.

20. Tanaka S. Cancer stem cells as therapeutic targets of hepato-biliary-pancreatic cancers. J Hepato-Biliary-Pancreat Sci 2015;22: 531-7.

21. Agostini M, Knight RA. miR-34: from bench to bedside. Oncotarget 2014;5:872-81.

22. Hage $\mathrm{C}$, Rausch V, Giese $\mathrm{N}$ et al. The novel c-Met inhibitor cabozantinib overcomes gemcitabine resistance and stem cell signaling in pancreatic cancer. Cell Death Dis 2013;4:e627.

23. Moriyama T, Ohuchida K, Mizumoto K, et al. MicroRNA-21 modu- lates biological functions of pancreatic cancer cells including their proliferation, invasion, and chemoresistance. Mol Cancer Ther 2009;8:1067-74.

24. Lu Y, Lu J, Li X et al. MiR-200a inhibits epithelial-mesenchymal transition of pancreatic cancer stem cell. BMC Cancer 2014;14:85.

25. Kloppel G, Mailler B. Histological typing of pancreatic and periampullary carcinoma. Eur J Surg Oncol 1991;17:139-52.

26. Hruban RH, Maitra A, Kern S, Goggins M. Precursors to pancreatic cancer. Gastroenterol Clin North Am 2007;36:831-49.

27. Murphy SJ, Hart SN, Lima JF, et al. Genetic alterations associated with progression from pancreatic intraepithelial neoplasia to invasive pancreatic tumor. Gastroenterology 2013;145:1098-109.

28. Hermanek P, Gall FP, Wittekind CH. Early-stage pancreatic ductal adenocarcinoma surgery. Int J Pancreatol 1994;16:302-4.

29. Nagakawa T, Kayahara M, Ueno K. A clinicopathological study on neural invasion in cancer of the pancreatic head. Cancer 1992; 69:930-5.

30. Tummala P, Junaidi O, Agarwal B. Imaging of pancreatic cancer: an overview. J Gastrointest Oncol 2011;2:168-74.

31. Gangi S, Fletcher JG, Nathan MA, et al. Time interval between abnormalities seen on, CT and the clinical diagnosis of PC: retrospective review of CT scans obtained before diagnosis. AJR Am J Roentgenol 2004;182:897-903.

32. Brais RJ, Davies SE, O'Donovan M, et al. Direct histological processing of EUS biopsies enables rapid molecular biomarker analysis for interventional pancreatic cancer trials. Pancreatology 2012; 12:8-15.

33. Tio TL, Tytgat GNJ, Cikot RJLM, et al. Ampullopancreatic carcinoma: preoperative TNM classification with endosonography. Radiology 1990;175:455-61.

34. Callery MP, Chang KJ, Fishman EK, et al. Pretreatment assessment of resectable and borderline resectable pancreatic cancer: expert consensus statement. Ann Surg Oncol 2009;16:1727-33.

35. Lygidakis NJ, van der Heyde MN, Lubbers MJ. Evaluation of preoperative biliary drainage in the surgical management of pancreatic head carcinoma. Acta Chir Scand 1987;153:665.

36. Benjamin IS. Benign and malignant lesions of the biliary tract. In: Carter D, Garden JO, Paterson-Brown S, eds. Hepato-biliary and pancreatic surgery. Philadelphia, PA: W B Saunders Company Ltd.; 1997. pp 201-258.

37. Pitt HA, Gomes AS, Lois JF. Does preoperative percutaneous biliary drainage reduce operative risk or increase hospital cost? Ann Surg 1985;201:405.

38. Warshaw AL, Tepper JE, Shipley WU. Laparoscopy in the staging and planning of therapy for pancreatic cancer. Am J Surg 1986; 151:76-9.

39. John TG, Greig JD, Carter DC, Garden OJ. Carcinoma of the pancreatic head and perimpullary region: tumour staging with laparoscopy, and laparoscopic ultrasonography. Ann Surg 1995;221: $136-64$.

40. Callery MP, Chang KJ, Fishman EK, et al. Pretreatment assessment of resectable and borderline resectable pancreatic cancer: expert consensus statement. Ann Surg Oncol 2009;16:1727-33.

41. Chun YS, Milestone BN, Watson JC, et al. Defining venous involvement in borderline resectable pancreatic cancer. Ann Surg Oncol 2010;17:2832-8.

42. Ishikawa 0, Ohigashi H, Sasaki Y, et al. Practical usefulness of lymphatic and connective tissue clearance for the carcinoma of the pancreas head. Ann Surg 1988;208:215.

43. Cameron JL, Crist DW, Sitzmann JV, et al. Factors influencing survival after pancreaticoduodenectomy for pancreatic cancer. Am J Surg 1991;161:120-5.

44. Campbell F, Smith RA, Whelan P, et al. Classification of R1 resections for pancreatic cancer: the prognostic relevance of tumour 
involvement within $1 \mathrm{~mm}$ of a resection margin. Histopathology 2009;55:277-83.

45. Esposito I, Kleeff J, Bergmann F, et al. Most pancreatic cancer resections are R1 resections. Ann Surg Oncol 2008;15:1651-60.

46. Birk D, Beger HG. Lymph-node dissection in pancreatic cancerWhat are the facts? Langenbecks Arch Surg 1999;384:158-66.

47. Sperling J, Schuld J, Hechler AM, Slotta JE, Kollmar 0. Extended versus standard lymphadenectomy in patients undergoing pancreaticoduodenectomy for periampullary adenocarcinoma: a prospective randomized single center trial. Eur Surg 2016;48:26-33.

48. Hidalgo M, Cascinu S, Kleef J, et al. Adresssing the challenges of pancreatic cancer: Future directions for improving outcome. Pancreatology 2015;15:8-18.

49. Neoptolemos JP, Stocken DD, Bassi C, et al. Adjuvant chemotherapy with fluorouracil plus folinic acid vs gemcitabine following pancreatic cancer resection: a randomized controlled trial. JAMA 2010;304:1073-81.

50. Boggi U, Napoli N, Costa F, et al. Robotic-assisted pancreatic resections. World J Surg 2016 [Epub ahead of print].

51. Fortner JG. Regional resection of cancer of the pancreas: a new surgical approach. Surgery 1973;73:307.

52. Buchler MW, Wagner M, Scmied BM, et al. Changes in morbidity after pancreatic resection: toward the end of completion pancreatectomy. Arch Surg 2003;138:1310-4; discussion 1315.

53. Wente MN, Buchler P, Friess H, Buchler MW. Adjuvant therapy in operable pancreatic cancer. Swiss Surg 2002;8:74-80.

54. Bramhall SR, Allum WH, Jones AG, et al. Treatment and survival in 13,560 patients with pancreatic cancer, and incidence of the disease, in the West Midlands: an epidemiological study. Br J Surg 1995;82:111-5.

55. Berrino F, De Angelis R, Sant M, et al. Survival for eight major cancers and all other cancers combined for European adults diagnosed in 1995-99: results of the EUROCARE-4 study. Lancet Oncol 2007;8:773-83.

56. van Heek NT, Kuhlmann KF, Scholten RJ, et al. Hospital volume and mortality after pancreatic resection: a systematic review and an evaluation of intervention in the Netherlands. Ann Surg 2005;242:781-8.

57. Ozaki H, Kinnoshita T, Kosuge T, et al. Effectiveness of multimodality treatment for resectable pancreatic cancer. Int J Pancreatol 1990;7:195-200.

58. Tsuchiya R, Noda T, Harada N, et al. Collective review of small carcinomas of the pancreas. Ann Surg 1986;203:77-81.

59. The Gastrointestinal Tumor Study group. Further evidence of effective adjuvant combined radiotherapy and chemotherapy following curative resection of pancreatic cancer. Cancer 1987;59: 2006-10.

60. Neoptolemos JP, Stocken DD, Friess H, et al. European study group for pancreatic cancer. A randomized trial of chemoradiotherapy and chemotherapy after resection of pancreatic cancer. $\mathrm{N}$ Eng $\mathrm{J}$ Med 2004;350:1200-10.

61. Beger HG, Gansauge F, Büchler MW, Link KH. Intraarterial adjuvant chemotherapy after pancreaticoduodenectomy for pancreatic cancer: significant reduction in occurrence of liver metastasis. World J Surg 1999;23:946-9.

62. Sohn TA, Yeo CJ, Cameron JL, et al. Resected adenocarcinoma of the pancreas-616 patients: results, outcomes, and prognostic indicators. J Gastrointest Surg 2000;4:567-79.

63. Gillen S, Schuster T, Meyer Zum Büschenfelde C, et al. Preoperative/neoadjuvant therapy in pancreatic cancer: a systematic review and meta-analysis of response and resection percentages. PLoS Med 2010;7:e1000267.

64. Portal A, Pernot S, Tougeron D, et al. Nab-paclitaxel plus gemcitabine for metastatic pancreatic adenocarcinoma after Folfirinox failure: an AGE0 prospective multicentre cohort. Br J Cancer 2015;113:989-95.

65. Von Hoff DD, Ervin T, Arena FP, et al. Increased survival in pancreatic cancer with nab-paclitaxel plus gemcitabine. N Engl J Med 2013;369:1691-703.

66. Delitto D, Luchurst CM, Black BS, et al. Oncologic and perioperative outcomes following selective application of laparoscopic pancreaticoduodenectomy for periampullary malignancies. J Gastrointest Surg 2016 [Epub ahead of print].

67. Osako M, Yonezawa S, Siddiki B, et al. Immunohistochemical study of mucin carbohydrates and core proteins in human pancreatic tumours. Cancer 1993;71:2191-9.

68. Watanapa P, Williamson RCN. Surgical palliation for pancreatic cancer: developments during the past two decades. Br J Surg 1992;79:8-20.

69. Saar MG, Cameron JL. Surgical management of unresectable carcinoma of the pancreas. Surgery 1982;91:123-33.

70. Smith AC, Dowsett JF, Russell RCG, et al. Randomised trial of endoscopic stenting versus surgical bypass in malignant low bile duct obstruction. Lancet 1994;344:1655-60.

71. Huibregste K, Carr-Locke DL, Cremer M, et al. Biliary stent occlusion - a problem solved with self-expanding stents? Endoscopy 1992;24:391-4.

72. Blamey SL, Fearon KCH, Gilmour WH, et al. Prediction of risk in biliary surgery. Br J Surg 1983;70:535-8.

73. Falconer JS, Fearon KCH, Ross JA, et al. The acute phase protein response and duration of survival in patients with pancreatic cancer. Cancer 1995;75:2077-82.

74. Colucci G, Giuliani F, Gebbia V, et al. Gemcitabine alone or with cisplatin for the treatment of patients with locally advanced and/or metastatic pancreatic carcinoma: a prospective, randomized phase III study of the Gruppo Oncologico dell'Italia Meridionale. Cancer 2002;94:902-10.

75. Trede M, Schwall G, Saeger HD. Survival after pancreatoduodenectomy. Ann Surgery 1990;211:447.

76. Brooks JR, Culebras JM. Cancer of the pancreas: palliative operation, Whipple procedure or total pancreatectomy. Am J Surg 1976;131:516.

77. Cubilla AC, Fortner J, Fitzgerald PJ. Lymph node involvement in carcinoma of the head of the pancreas area. Cancer 1978;41:880.

78. Poston GJ, Gillespie J, Guillou PJ. Biology of pancreatic cancer. Gut 1991;32:800.

79. Carpelan-Holmstrom M, Nordling S, Pukkala E, et al. Does anyone survive pancreatic ductal adenocarcinoma? A nationwide study re-evaluating the data of the Finnish Cancer Registry. Gut 2005;54:385-7.

80. Crile G Jr, Isbister WH, Hawk WA. Carcinoma of the ampulla of Vata and the terminal bile and pancreatic ducts. Surg Gynec Obstet 1970;131:1052-4.

81. Nagal H, Kuroda A, Morioka Y. Lymphatic and local spreading of T1 and T2 pancreatic cancer. Ann Surg 1986;204:65.

82. Pour PM, Egami H, Takiyama Y. Patterns of growth and metastases of induced pancreatic cancer in relation to the prognosis and and its clinical implications. Gastroenterology 1991;100:529.

83. Nagakawa T, Mori K, Nakano T, et al. Perineural invasion of carcinoma of the pancreas and biliary tract. Br J Surg 1993;80:619.

84. Weledji EP, Assob JC. The ubiquitous Neural cell adhesion molecule. Ann Med Surg Ann Med Surg (Lond) 2014;3:77-81.

85. Kleeff J, Beckhove P, Esposito I, et al. Pancreatic cancer microenvironment. Int J Cancer 2007;121:699-705.

86. Satake K, Chung Y-S, Umeyama K, et al. The possibility of diagnosing small pancreatic cancer (less than $4.0 \mathrm{~cm}$ ) by measuring various serum tumour markers. Cancer 1991;68:149-52.

87. Staabb H, Brummerdorf T, Hornung A, et al. The clinical validity of circulating tumor-associated antigens CEA and CA 19-9 in primary 
diagnosis and follow-up of patients with gastrointestinal malignancies. Klin Wschr 1985;63:106-15.

88. Tian F, Appert H, Myles J, Howard J. Prognostic value of serum CA 199 levels in pancreatic adenocarcinoma. Ann Surg 1992;215:350-5.

89. Macgregor-Das AM, Iacobuzio-Donahue CA. Molecular pathways in pancreatic carcinogenesis. J Surg Oncol 2013;107:8-14.

90. Eser S, Reiff N, Messer M, et al. Selective requirement of PI3K/PDK1 signaling for Kras oncogene-driven pancreatic cell plasticity and cancer. Cancer Cell 2013;23:406-20.

91. Jones S, Zhang X, Parsons DW, et al. Core signaling pathways in human pancreatic cancers revealed by global genomic analyses. Science 2008;321:1801-6.

92. Moore MJ, Goldstein D, Hamm J, et al. Erlotinib plus gemcitabine compared with gemcitabine alone in patients with advanced pancreatic cancer: a phase III trial of the National Cancer Institute of Canada Clinical Trials Group. J Clin Oncol 2007;25:1960-6.
93. Chari ST. Detecting early pancreatic cancer: problems and prospects. Semin Oncol 2007;284-94.

94. Kaur S, Bane MJ, Jain M, et al. Early diagnosis of pancreatic cancer: challenges and new developments. Biomark Med 2012;6:597-612.

95. Melo SA, Luecke LB, Kahlert C, et al. Glypican-1 identifies cancer exosomes and detects early pancreatic cancer. Nature 2015; 523:177-82.

96. Zhang Y, Choi M. Immune therapy in pancreatic cancer: now and the future? Rev Recent Clin Trials 2015;10:317-25.

97. Tanemura M, Miyoshi E, Nagano H, et al. Cancer immunotherapy for pancreatic cancer utilizing $\alpha$-gal epitope/natural anti-Gal antibody reaction. World J Gastroenterol 2015;40:11396-410.

98. Collignon A, Perles-Barbacaru AT, Robert S, et al. A pancreatic tumor-specific biomarker characterized in humans and mice as an immunogenic onco-glycoprotein is efficient in dendritic cell vaccination. Oncotarget 2015;6:23462-79. 